\title{
Experimental Investigations of the Radio Wave Attenuation Processes Through the Paths Leaving Buildings
}

\author{
Oleg V. Chernoyarov ${ }^{1,2^{*}}$, Boris V. Matveev ${ }^{3}$ and Alexey A. Artemenko ${ }^{4}$ \\ ${ }^{1}$ Russia, 111250, Moscow, Krasnokazarmennaya st., 14, National Research University “Moscow Power Engineering Institute” \\ ${ }^{2}$ Russia, 634050, Tomsk, Lenin Avenue, 36, International Laboratory of Statistics of Stochastic Processes and Quantitative \\ Finance of National Research Tomsk State University \\ ${ }^{3}$ Russia, 394026, Voronezh, Moscow Avenue, 14, Voronezh State Technical University \\ ${ }^{4}$ Russia, 394018, Voronezh, Universitetskaya sq., 1, Voronezh State University \\ ${ }^{*}$ Corresponding author
}

\begin{abstract}
We consider the problem of the determination of the radio wave attenuation factor. We show that the attenuation factor can be used for the information leakage detecting due to the side electromagnetic radiation of information facilities. We illustrate the thematic examples of the experimental determination of the attenuation factor for decimetric waves propagating through the paths leaving buildings. We give references for obtaining of the correct results during simulation of radio wave attenuation processes for the paths leaving buildings.
\end{abstract}

Keywords-radio wave attenuation; side electromagnetic radiation; interception

\section{INTRODUCTION}

Determination of radio wave attenuation in city conditions is an important scientific and technical problem. Its decision simplifies the communication system design, the overcoming of electromagnetic compatibility problems and the information leakage detecting due to the side electromagnetic radiation [1]. The last of the problems mentioned above is of interest, as the use of known information security ways can result in big shielding costs or in some shortcomings of noise masking facilities. Need of application of some or other security facilities is defined by the conducting of special studies on possibility of information interception from premises, in which the data processing is carried out by hardware (for example, computers).

Natural (simplest) way of the task solution of the determination of radio-wave attenuation parameters is metering on object. However, such metering is labor-intensive process. Besides, there is essential feature for this task: measurements are made within "small" distances (tens and hundreds of meters). But, as it can be seen from the task solution of the radio-wave propagation over an earth surface, the monotonous law of field attenuation (Vvedensky formula) is valid for "long" distances only. For "small" distances the attenuation law has nonmonotonic character with moving off from a source [2]. The similar pattern of the non-monotony field attenuation is also reproduced in frequency domain. For radio scattering in city conditions the attenuation law and frequency dependences become complicated considerably. Therefore, separate experimental samples cannot give the unequivocal information on side electromagnetic radiation attenuation.

\section{AtTENUATION FACTOR ANALYSIS}

The attenuation factor $K(r, d, f)$ for frequency $\mathrm{f}$ is determined as the ratio of electric field intensity $E(r, f)$ on a distance $r$ to the intensity $E(d, f)$ in a reference point on a distance d: $K(r, d, f)=E(r, f) / E(d, f)$ and it is used for estimation of the information security due to side electromagnetic radiation leakage [1].

We have conducted the series of measurements for two paths. In both cases the radiating device was placed in one of the building rooms on the second floor. Received intensity was preliminary measured in a reference point on distance of 1 meter from a source. All other measurements were made outside of the building on distances 5, 10 and 20 meters from a source accordingly. The first path was laid in a direction of the window in the building (semi-closed path). And the second path was laid in a wall direction (closed path). For each path the measurements were made within two bands: $0.3 \ldots 1$ and 1 ...3 GHz for each measuring (check) point (5, 10, 20 meters). We applied a nondirectlonal receiving antenna (dipole) for the lower band and directional (horn) antenna for the upper band. The results of these measurements are presented by curves of frequency dependences $E(f)=\left.E(r, f)\right|_{r=\text { const }}$ in Figures 1, 2. 

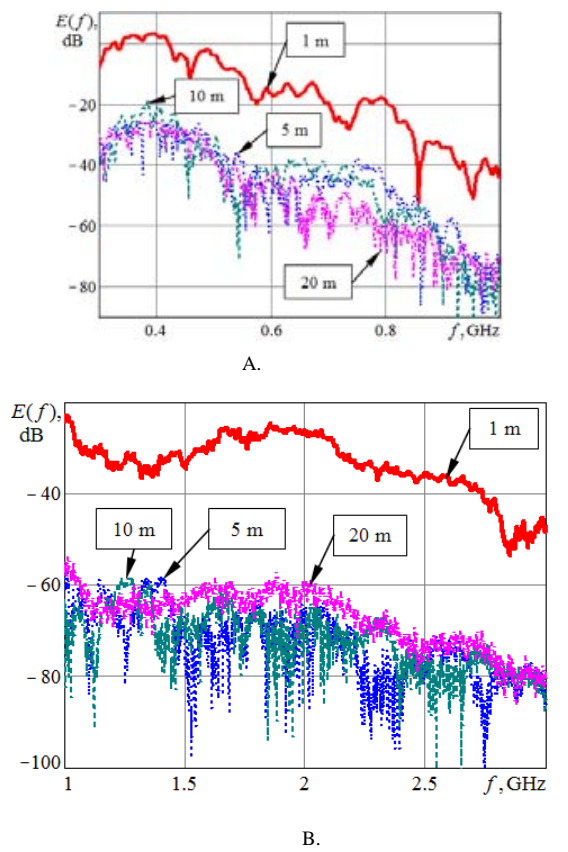

FIGURE I. THE FREQUENCY DEPENDENCES OF THE RECEIVING ELECTRIC FIELD INTENSITY FOR CHECK POINTS ON THE CLOSED PATH WITHIN BAND: A) $0.3 \ldots 1 \mathrm{GHZ}$, B) $1 \ldots .3 \mathrm{GHZ}$

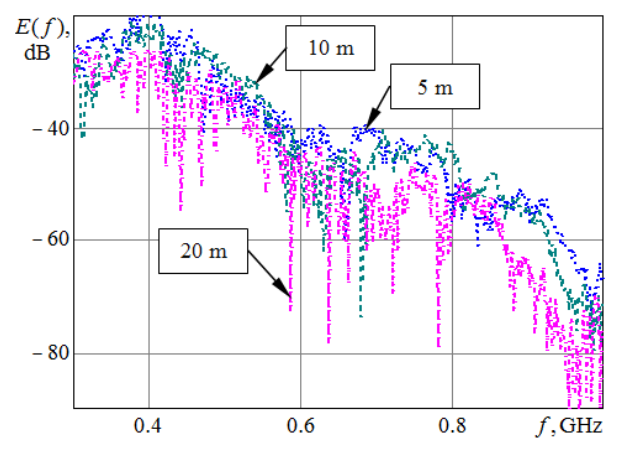

A.

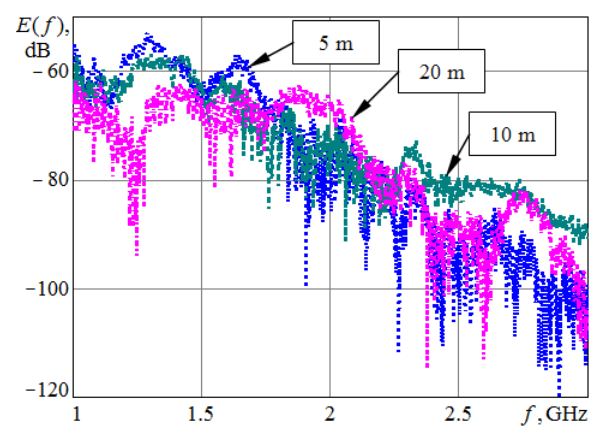

B.

FIGURE II. THE FREQUENCY DEPENDENCES OF THE RECEIVING ELECTRIC FIELD INTENSITY FOR CHECK POINTS ON THE CLOSED PATH WITHIN BAND: A) $0.3 \ldots 1 \mathrm{GHZ}$, B) $1 \ldots 3 \mathrm{GHZ}$

In Figures 1, 2 we can see some features of radio-wave propagation through the paths leaving buildings. Firstly, the effect of the nonmonotonic attenuation reveals itself clearly. In other words, for any selected measuring point $(5,10,20$ meters) there is a frequency band in which the magnitude of received signal will be more, than in all other points. For example, as follows from Figure 1b, there are frequency bands (in particular, 1.5-2.5 GHz), where the best reception occurs in more distant point (20 meters).

Such effect of the nonmonotonic attenuation can be observed with other curves in Figures 1 and 2, but it is less pronounced. Secondly, for all dependences (Figures 1, 2) the correlation caused by the amplitude-frequency response of measurement instrumentation is characteristic. This correlation reveals itself clearly for a semi-closed path (Figure 1b) with measuring points of 1 and 20 meters that also it is corroborated by calculations of the correlation coefficient [3] (see Table I).

TABLE I. THE CORRELATION COEFFICIENT OF THE FREQUENCY DEPENDENCES OF THE ELECTRIC FIELD INTENSITY BETWEEN A REFERENCE POINT (1 METER) AND CHECK POINTS IN BAND OF $1 \ldots 3$ GHZ

\begin{tabular}{|l|c|c|c|}
\hline Distance, m & 5 & 10 & 20 \\
\hline Semi-closed path & 0.53 & 0.54 & 0.91 \\
\hline Closed path & 0.69 & 0.58 & 0.78 \\
\hline
\end{tabular}

In a frequency band of $0.31 \mathrm{GHz}$ the high correlation is observed between dependences for all points. It means that for frequencies below $1 \mathrm{GHz}$ the absorption of radio waves into the wall of the building is insignificant, and ripple of frequency response of electric field intensity is caused by resonant effects into walls. Thirdly, each of frequency dependences contains the extrema caused by a radio wave interference. At determining the attenuation factor according to the formula (1), the interference minima and maxima of frequency dependence of the electric field intensity occur also (Figure 3).

From the above-stated it is possible to conclude a number of recommendations for an estimation of the information security from leakage due to the side electromagnetic radiation. Minima in frequency domain are not informative, since they come out of considerable radio-wave attenuation caused by multipath propagation. And so such measurements should be rejected. It is achieved by smoothing of experimentally obtained dependences of electric intensity in frequency domain with the use of various procedures. The positive interference causing the maxima of electric intensity does not also increase the probability of reception of the information transferred by the side electromagnetic radiation as it, as a rule, leads to distortions of the information pulses. Therefore, it is necessary to aspire to a single-beam receive mode by attenuation factor measurement.

The use of smoothing allows us to avoid the attenuation factor deflation, as in work [4]. We establish that the Gaussian filter can be effectively applied for smoothing. In Figure 3 the frequency dependences of the attenuation factor constructed applying the Gaussian filter with bandwidth $15 \mathrm{MHz}$ (for lower frequency band) and $30 \mathrm{MHz}$ (for upper frequency band) are represented. 

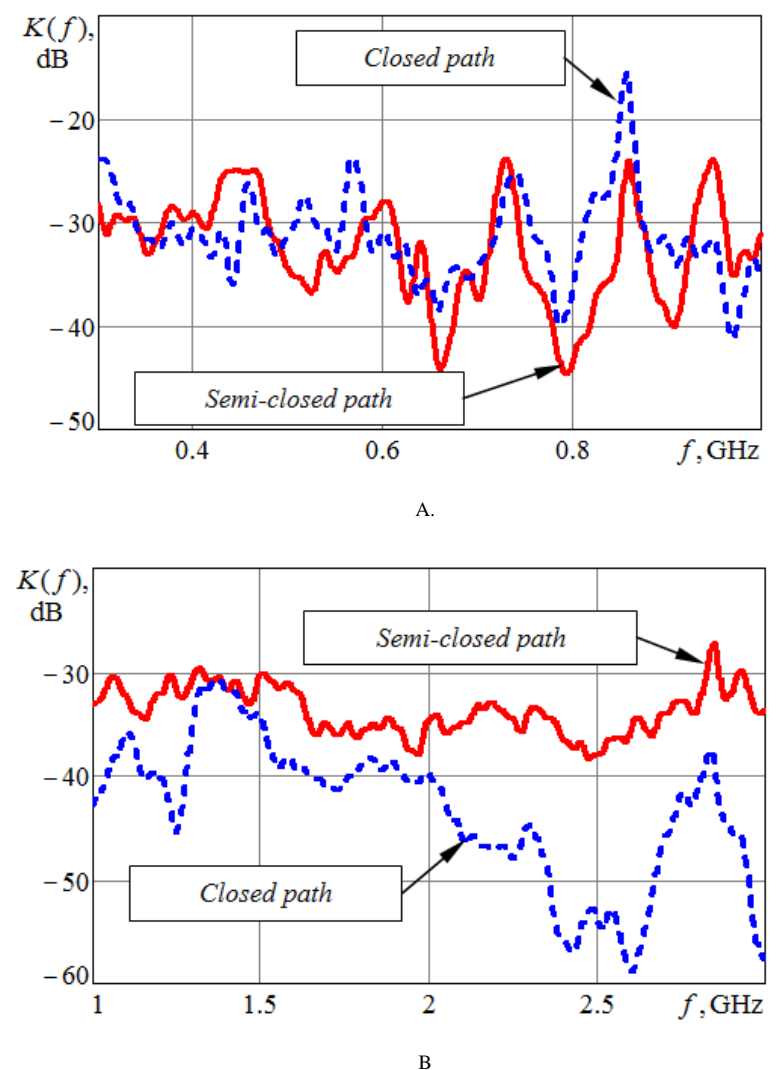

FIGURE III. THE SMOOTHED ATTENUATION FACTOR IN THE CHECK POINT OF 20 M FOR THE CLOSED AND SEMI-CLOSED PATHS IN BAND: A) $0.3 \ldots 1 \mathrm{GHZ}, \mathrm{B}) 1 \ldots 3 \mathrm{GHZ}$

In practice, in order to disclose the paths with the least attenuation, and therefore, the most preferable to the side electromagnetic radiation reception the simulation based on geometrical optics approximation [5] can be used. Here the field in a reception point is calculated as the vector sum of the rays scattered by all possible obstacles [3]. And also it is presupposed that receiving and transmitting antennas are point sources located in a far-field region for each other.

It should be noted that the use of the ray approximation for simulation is not capable to reproduce the features of the radio scattering caused by large count of small objects. In this case the simulation results should be compared with the experimental results processed by smoothing (for example, Gaussian) filters and similar to shown in Figure 3.

\section{CONCLUSION}

While determining the possibility of side electromagnetic radiation reception, it is necessary to take into account the strong multipath radio-wave propagation influence on measured results. Measurements should be conducted not only at frequencies of test signal harmonic components, as it is suggested in [1], but also in a wide frequency range with small step. The experimental data should be additionally processed, for example, with the smoothing within a frequency band comparable to a frequency band of signal reception.

\section{ACKNOWLEDGMENT}

The reported study was supported by Russian Science Foundation (research project No. 15-11-10022)

\section{REFERENCES}

[1] G.A. Buzov, S.V. Kalinin, A.V. Kondrat'ev, Leak Protection through Technical Channels [in Russian], Goryachaya Liniya-Telekom, Moscow, 2005.

[2] M.P. Dolukhanov, Radio-wave Propagation [in Russian], Svyaz', Moscow, 1972.

[3] V.I. Tikhonov, V.N. Kharisov, Statistical Analysis and Synthesis of Radio Engineering Devices and Systems [in Russian], Radio i Svyaz', 2004.

[4] A.N. Katrusha, "Research of attenuation of radio waves of the range 100 $\mathrm{MHz}-2 \mathrm{GHz}$ on the path of propagation from the building on street", Journal of Radio Electronics, No 10, 2013, pp. 1-12 (http://jre.cplire.ru/jre/oct13/14/text.html).

[5] D.V. Asotov, B.V. Matveev, O.V. Chernoyarov, E.A. Lysina, "Radio Waves Attenuation Model for a Ray Approximation”, 2015 International Siberian Conference on Control and Communications (SIBCON),

[6] Conference Proceedings, Omsk, 2015, pp. 1-5. 